\title{
Avaliação de inseticidas no controle de Sitophilus oryzae (Linnaeus) (Coleoptera, Curculionidae) e Rhyzopertha dominica (Fabricius) (Coleoptera, Bostrichidae) em trigo armazenado ${ }^{1}$
}

\author{
Rui S. Furiatti ${ }^{2}$ \\ Paulo R.V.S. Pereira ${ }^{3}$ \\ Airton R. Pinto Jr. ${ }^{3}$ \\ Flávio A. Lazzari ${ }^{3}$
}

\begin{abstract}
Efficacy of insecticides to control Sitophilus oryzae (Linnaeus) (Coleoptera, Curculionidae) and Rhyzopertha dominica (Fabricius) (Coleoptera, Bostrichidae) in stored wheat. The efficiency of insecticides against Sitophilus oryzae (Linnaeus, 1763) (Coleoptera, Curculionidae) and Rhyzopertha dominica (Fabricius, 1792) (Coleoptera, Bostrichidae) was determined exposing them to treated wheat in laboratory. Each plot, which was composed by $1.2 \mathrm{~kg}$ of wheat grain, was treated with $5 \mathrm{ml}$ of the concentration $/ \mathrm{kg}$ of wheat using a propelling air atomizer. Fifteen days after treatment $50 \mathrm{~g}$ samples of the grain were taken, from each plot, placed in plastic jars into which 10 adult insects of each species were released. This was repeated every 30 days until 165 days after treatment. The number of dead insects was counted 15 days after each infestation thus the evaluations were made at 30,60 , $90,120,150$ and 180 days after the insecticide application. Esfenvalerate+fenitrothion+pyperonil butoxide $(0,375+7,5+3,0$ and $0,5+10,0+4,0 \mathrm{~g}$ a.i. $/ \mathrm{t})$, fenitrothion+esfenvalerate $(7,5+0,375$ and $10,0+0,375 \mathrm{~g}$ a.i./t), fenitrothion+synergised deltamethrin $(7,5+0,375 \mathrm{~g}$ a.i./t $)$ and fenitrothion $(7,5 \mathrm{~g}$ a.i./t) were effective against Sitophilus oryzae until 180 days after treatment. Esfenvalerate+fenitrothion+pyperonil butoxide $(0,5+10,0+4,0 \mathrm{~g}$ a.i./t $)$, fenitrothion+esfenvalerate $(7,5+0,375$ and $10,0+0,375 \mathrm{~g}$ a.i./t $)$, fenitrothion+synergised deltamethrin $(7,5+0,375 \mathrm{~g}$ a.i./t) were effective against $R$. dominica until 180 days after treatment. Synergised deltamethrin $(0,375 \mathrm{~g}$ a.i./t) was effective against $S$. oryzae until 150 days and against $R$. dominica until 120 days after treatment. Esfenvalerate $(0,5 \mathrm{~g}$ a.i. $/ \mathrm{t})$ was ineffective against $S$. oryzae and effective until 60 days after treatment against $R$. dominica. Fenitrothion $(7,5 \mathrm{~g}$ a.i./t) was ineffective against $R$. dominica. The use of mixtures of organophosphorus plus pyrethroids were more effective against $S$. oryzae and $R$. dominica when compared with these insecticides used alone.
\end{abstract}

KEY WORDS. Insecta, chemical control, stored-product pests

A cultura do trigo, no Brasil e a nível mundial, sempre mereceu destaque, tratando-se do mais importante cereal cultivado pelo homem, principalmente, devido à sua ampla utilização na alimentação humana (SCHEEREN 1986).

1) Contribuição número 917 do Departamento de Zoologia, Universidade Federal do Paraná.

2) Departamento de Agronomia, Universidade Estadual de Ponta Grossa. Praça Santos Andrade, Caixa Postal 992, 84100-970 Ponta Grossa, Paraná, Brasil.

3) Departamento de Zoologia, Universidade Federal do Paraná. Caixa Postal 19020, 81531-990 Curitiba, Paraná, Brasil. 
ALMEIDA (1989) estima que as perdas quantitativas anuais causadas por pragas durante o período de armazenamento de grãos são da ordem de $10 \%$ da produção mundial. Esta estimativa refere-se, apenas, ao ataque dos insetos ao embrião e ao endosperma das sementes, não entrando em consideração o aquecimento da massa de grãos provocada pela atividade dos insetos, o conseqüente ataque de fungos e também, a diminuição do valor nutritivo dos grãos.

No Brasil as perdas anuais pelo ataque de pragas chegam a $10 \%$ da produção armazenada. A baixa disponibilidade de inseticidas registrados para o controle de pragas de produtos armazenados contribui para o agravamento deste problema, uma vez que nem todos os produtos podem ser utilizados para todas as pragas e nas diversas situações de armazenamento encontradas no Brasil (LORINI \& SCHNEIDER 1994). Dentre as pragas que atacam grãos de trigo durante o armazenamento destacam-se Sitophilus zeamais (Motschusky, 1885), S. oryzae (Linnaeus, 1763) (Coleoptera, Curculionidae) e Rhyzopertha dominica (Fabricius, 1792) (Coleoptera, Bostrichidae), sendo considerados pragas primárias por terem a capacidade de perfurar o tegumento de sementes e grãos.

Os principais inseticidas usados para o tratamento de grãos armazenados são os organofosforados malatiom, pirimifós-metílico, fenitrotiom, diclorvós e os piretróides deltametrina e permetrina.

Este trabalho teve por objetivo avaliar a eficiência de inseticidas no controle de $S$. oryzae e $R$. dominica em trigo armazenado.

\section{MATERIAL E MÉTODOS}

O experimento foi realizado no Departamento de Zoologia da Universidade Federal do Paraná, onde foram mantidas em sala climatizada $\left(25 \pm 2^{\circ} \mathrm{C}\right)$ as colônias de $S$. oryzae e de $R$. dominica, provenientes de diversos pontos do Estado do Paraná usadas neste experimento. $\mathrm{O}$ delineamento estatístico utilizado foi de blocos casualizados, com quatro repetições.

Cada parcela foi constituída de $1,2 \mathrm{~kg}$ de trigo, que após tratados, foram acondicionados em sacos de ráfia e armazenados em sala climatizada $\left(25 \pm 2^{\circ} \mathrm{C}\right)$.

A aplicação dos inseticidas foi efetuada com um pulverizador manual propulsionado a ar, equipado com bico pneumático com vazão de 5,0 l/t. Cada parcela recebeu $5 \mathrm{ml}$ de calda por kg de grãos de trigo e na testemunha apenas água. Os inseticidas foram aplicados dentro de sacos plásticos de $0,4 \times 0,5 \mathrm{~m}$ e homogeneizados por dois minutos.

Os inseticidas usados foram: esfenvalerate+butóxido de piperonila a $0,25+2,22$ e $0,5+4,44$ g i.a./t; esfenvalerate (Sumidan $\left.{ }^{(}\right) 25 \mathrm{CE}$ ) a $0,5 \mathrm{~g}$ i.a. $/ \mathrm{t}$; esfenvalerate+fenitrotiom+butóxido de piperonila a $0,375+7,5+3,0$ e $0,5+10,0+4,0$ $\mathrm{g}$ i.a./t; fenitrotiom+esfenvalerate a 7,5+0,375 e 10,0+0,5 g i.a./t; fenitrotiom+deltametrina a $7,5+0,375$ g i.a./t; fenitrotiom (Sumigran ${ }^{(B)} 500 \mathrm{CE}$ ) a 7,5 g i.a./t e deltametrina (k-Obiol ${ }^{\circledR} 25 \mathrm{CE}$ ) a 0,375 g i.a./t. A formulação K-Obiol ${ }^{\circledR} 25 \mathrm{CE}$ possui butóxido de piperonila proporção de 10:1 em relação a deltametrina.

Para a avaliação da eficiência, em intervalos de 15 dias após a aplicação dos inseticidas, $50 \mathrm{~g}$ de cada parcela, foram infestadas com 10 insetos adultos, não sexados e sem padronização de idade das espécies testadas, acondicionadas em copos plásticos de $0,5 \mathrm{I} \mathrm{em} \mathrm{sala} \mathrm{climatizada}\left(25 \pm 2^{\circ} \mathrm{C}\right)$. A avaliação do número de 
insetos mortos foi feita 15 dias após cada infestação, ou seja, aos 30, 60, 90, 120, 150 e 180 dias após a aplicação dos inseticidas.

Considerou-se eficiente o inseticida que não apresentou diferença estatística a $100 \%$ de mortalidade de $S$. oryzae e $R$. dominica, e diferença estatística significativa da testemunha.

O número de insetos mortos por parcela foi submetido a análise de variância e as diferenças entre as médias discriminadas pelo teste de Tukey a $5 \%$. A eficiência relativa dos inseticidas foi calculada pela fórmula de Abbot (АВвотт 1925).

\section{RESULTADOS E DISCUSSÃO}

No controle de Sitophilus oryzae, o inseticida esfenvalerate $(0,5 \mathrm{~g}$ i.a./t) e a mistura deste com o sinergista butóxido de piperonila (BPO) nas dosagens de $0,25+2,22$ e $0,5+4,44 \mathrm{~g}$ i.a./t foram ineficientes desde o início do experimento. A deltametrina $(0,375 \mathrm{~g}$ i.a./t) foi eficiente até os 150 dias após a aplicação. Os demais inseticidas, esfenvalerate + fenitrotiom $+\mathrm{BPO}(15$ e $20 \mathrm{ml} / \mathrm{t})$, fenitrotiom+esfenvalerate $(7,5+0,375$ e $10,0+0,5$ g i.a./t), fenitrotiom+deltametrina $(7,5+0,375$ g i.a./t) e fenitrotiom ( $7,5 \mathrm{~g}$ i.a./t) foram eficientes até os 180 dias quando o experimento foi encerrado (Tab. I).

YADAV (1988), obteve resultados semelhantes onde a deltametrina mostrouse eficiente contra $S$. oryzae por um período de 120 a 170 dias. Também, YADAV \& SiNGH (1994) observaram proteção da deltametrina por um período de 180 dias. Entretanto BITRAN et al. (1991), não observaram uma ação residual prolongada de deltametrina contra $S$. oryzae. SHRIVASTAVA et al. (1991) testaram fenitrotiom em várias espécies de insetos de produtos armazenados, sendo este eficiente apenas para $S$. oryzae, semelhante a resultados quando usado isolado ou em mistura.

No controle de $R$. dominica, o inseticida fenitrotiom (7,5 g i.a./t) foi ineficiente desde o início do experimento, porém não diferiu estatisticamente dos demais inseticidas aos 60 e 120 dias após a aplicação. Esfenvalerate $(0,5 \mathrm{~g}$ i.a./t), esfenvalerate+BPO $(0,25+2,22$ e $0,5+4,44$ g i.a./t $)$ e esfenvalerate+fenitrotiom + BPO $(0,375+7,5+3,0 \mathrm{~g}$ i.a./t $)$ e a deltametrina $(0,375 \mathrm{~g}$ i.a./t $)$ apresentaram eficiência até os 120 dias após o tratamento. Os demais tratamentos, esfenvalerate+fenitrotiom+BPO $(0,5+10,0+4,0 \mathrm{~g}$ i.a./t $)$, fenitrotiom+esfenvalerate $(7,5+0,375$ e $10,0+0,5$ g i.a./t), fenitrotiom+deltametrina $(7,5+0,375 \mathrm{~g}$ i.a./t), foram eficientes até 180 dias após a aplicação dos inseticidas (Tab. II).

A proteção demonstrada pela deltametrina contra $R$. dominica está em desacordo com YADAV (1988) e YADAV \& SING (1994), onde foi observado que o período de proteção ao inseto durou 180 dias, contra os 150 dias observados neste experimento. Corroborando com dados obtidos neste experimento BARETH \& GUPTA (1989a), ISLAM et al. (1989) e SCHIFFERS et al. (1989), obtiveram proteção até os 150 dias após os tratamentos com deltametrina. KoRUNIC et al. (1985) obtiveram resultados semelhantes a este trabalho, ao comprovarem que deltametrina utilizada em conjunto com butóxido de piperonila foi eficiente no controle de $S$. oryzae e $R$. dominica, proporcionando uma proteção de 168 dias após a aplicação dos inseticidas. 

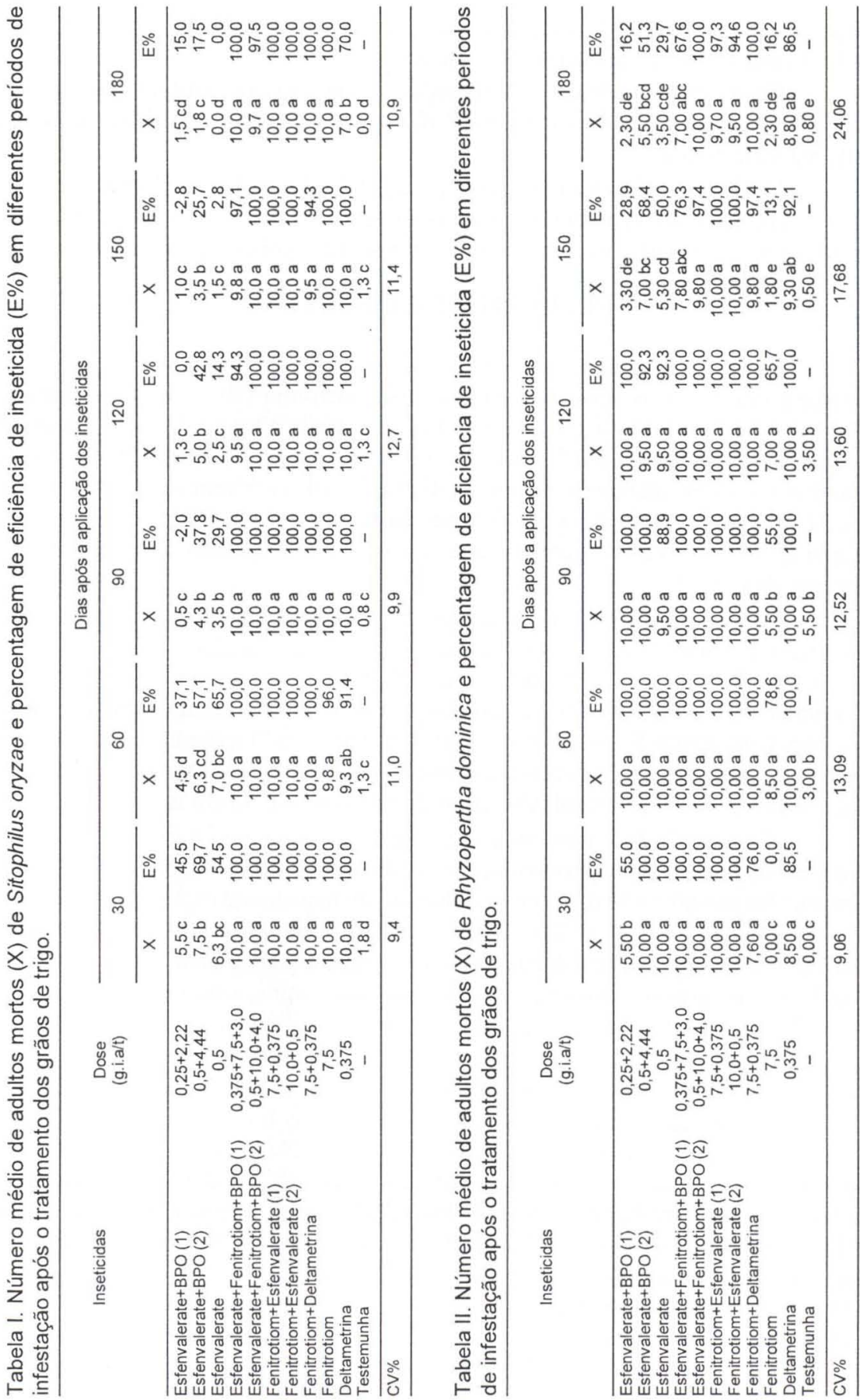

Revta bras. Zool. 16 (1): 221 - 226, 1999 
A ineficiência de fenitrotiom contra $R$. dominica é confirmada por BITRAN et al. (1991), entretanto DESMARCHELIER et al. (1987) e BARETH \& GUPTA (1989b), observaram que este inseticida foi eficiente contra este inseto.

Foram eficientes para $S$. oryzae e $R$. dominica, até os 180 dias após a aplicação, os inseticidas esfenvalerate+fenitrotiom+BPO $(0,5+10,0+4,0$ g i.a./t), fenitrotiom+esfenvalerate $(7,5+0,375$ e $10,0+0,5$ g i.a./t $)$ e fenitrotiom+deltametrina $(7,5+0,375$ g i.a. $/ \mathrm{t})$.

Os tratamentos compostos por misturas de organofosforados e piretróides foram os que apresentaram os melhores resultados para as duas pragas. Estes mesmos produtos quando utilizados isoladamente mostraram eficiência para uma ou outra praga, mas não para ambas. Os resultados obtidos neste trabalho poderão ser empregados no controle de pragas de produtos armazenados, entretanto, deve-se salientar que o uso de misturas de inseticidas deve ser estudado criteriosamente, visto que poderão surgir problemas com as misturas, como a resistência aos inseticidas envolvidos.

\section{REFERÊNCIAS BIBLIOGRÁFICAS}

AввотT, W.S. 1925. A method of computing the effectiveness of an insecticide. Jour. Econ. Entomol. 18: 265-267.

BARETH, S.S. \& H.C. GUPTA. 1989a. Efficacy of gunny bag and seed treatment on the natural infestation of Rhyzopertha dominica on stored wheat. Seed Res. 17: 178-181.

ALMEIDA, A.A. 1989. Natureza dos danos causados por insetos em grãos armazenados. Anais XI do Congresso Brasileiro de Entomologia, Campinas, 4: 16-32.

1989b. Efficacy of six insecticides for the protection of stored wheat seeds against Rhyzopertha dominica (Fab.) Seed Res. 17: 43-46.

Bitran, E.A.; T.B. Campos; N. Suplicy Filho \& S. ChiBa. 1991. Evaluation of the residual action of some insecticides on the protection of maize, wheat and rice grains against stored products pests. Arq. Inst. Biol., São Paulo, 58 (1-2): 43-50.

Desmarchelier, J.M.; M. Bengston; R. Davies; B. Elder; R. Hart; R. HenninG; W. Murray; E. Ridley; E. RipP; C. SierakowsKi; R. Sticka; J. SNELSON; B. WALLBANK \& A. WilSON. 1987. Assessment of the grain protectants chlorpyrifos-methyl plus bioresmetrin, fenitrothion plus (1R)-phenothrin, metacrifos and pirimifhos-methyl plus carbaryl under practical conditions in Australia. Pest. Sc. 20: 271-288.

Islam, N.; M.I.M. Bhuiyah; A. Begum \& M.A. Karim. 1989. Comparative efficacy of different material against Sitophilus oryzae L. infesting maize seeds in storage. Bangl. Jour. Z. 17: 175-178.

KorUnic, Z.; D. HAMEL-KorEN \& K. D-HAMEL. 1985. The effect of deltamethrin and pirimiphos-methyl on pests of stored products. Zastita-Bilja 36 (4): 417 423.

LORINI, I. \& S. SCHNEIDER. 1994. Pragas de Grãos Armazenados: resultados de pesquisa. Passo Fundo, Embrapa-CNPT, 48p. 
SCHEEREN, P.L. 1986. Informações sobre o trigo Triticum spp. Passo Fundo, Empresa Brasileira de Pesquisa Agropecuária, Centro Nacional de Pesquisa do Trigo, 34p.

Schiffers, B.C.; E. Haubruge; E. Gabriel; C. Rodriguez-Cobos \& J.M. LEDEINE. 1989. The comparative toxicity of five pyrethroid insecticides to six insect pests of stored products. Med. Van de Facul. Landb. Rijksun. Gent. 54 (3b): 1095-1103.

Shrivastava, A.P.; K. NARAIN \& P.D. Bhandari. 1991. Evaluation and assessment of newly reported insecticides for control of insect pests of stored products. Entomology for defense services: 219-225.

YADAV, T.D. 1988. Efficacy of deltamethrin against storage insects in rice and wheat under FCI's storage system. Pest. 22: 39-43.

YADAV, T.D. \& S. SINGH. 1994. Persistence toxicity and efficacy of four insecticides as jute fabric treatment to protect cereal and legume seeds. I. Jour. Entomol. 56 (2): 146-155.

Recebido em 07.XI.1997; aceito em 08.III.1999. 